
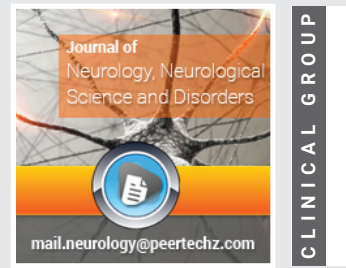

\title{
Epilepsy: From pediatric to adulthood. Definition, classifications, neurobiological profiles and clinical
}

\section{ISSN: $2641-2950$ \\ Received: 29 June, 2020 \\ Accepted: 21 July, 2020}

Published: 22 July, 2020

*Corresponding author: Dr. Giulio Perrotta, Psychologist sp.ed Strategic Psychotherapist, Forensic Criminologist, Jurist sp.ing SSPL, Lecturer, Essayist, Italy E-mail: giuliosr1984@hotmail.it

ORCID: https://orcid.org/0000-0003-0229-5562 https://www.peertechz.com

Check for updates

\section{treatments}

\section{Giulio Perrotta*}

Psychologist sp.ed Strategic Psychotherapist, Forensic Criminologist, Jurist sp.ing SSPL, Lecturer,

Essayist, Italy

\begin{abstract}
This work focuses on the specific analysis of the general, neurobiological and clinical profiles of epileptic forms, starting from classifications and definitions, and then extending the field of study to clinical and symptomatological data, to conclude with treatments and better management of these patients.
\end{abstract}

\section{Contents of the manuscript}

Introduction: Definition, classifications and symptomatic profiles

Introduction and definition: "Epilepsy" - from the Greek $\dot{\varepsilon} \pi \imath \eta \psi \psi^{\prime} \alpha$, "to be caught, hit by something" - [1], is a neurological condition characterized by recurrent and sudden (at least two twenty-four hours apart) [2], physical manifestations of sudden loss of consciousness and violent convulsive movements of the muscles (the so-called "epileptic seizures") $[3,4]$. We will, therefore, speak of "epilepsy" only when the cause of the seizure will be primary (and not secondary); in all other cases, in the clinical setting it is preferable to speak of "epileptic seizure" [5,6].

In ancient times, epilepsy was associated with religious experiences and demonic or divine possession. Known as the "sacred disease", it was widely described in the fifth century BC. by Hippocrates of Kos, since epileptic seizures were thought to be a form of attack by demons, or that the visions experienced by patients were messages from the gods; the father of modern medicine himself, however, raised doubts about the divine nature of the phenomenon. However, this belief was more realistic and clinical in the Indian area, where there was already talk of "loss of consciousness". However, in most ancient cultures (and some modern cultures, in Africa and Asia), people with epilepsy were stigmatized, avoided, or even imprisoned, because they were considered dangerous, contagious, or cursed [7].

\section{Etiological and statistical profiles}

In the epilepsy hypothesis, the etiology is not known and the hypotheses can be multiple; the etiologies do not have a hierarchical order and the importance attributed to the etiological group in which the patient is framed can depend on the circumstance [8]:

a) Structural etiology: A structural etiology refers to the presence of morphological anomalies visible to neuroimaging where the electroclinical study and the finding of neuroimaging are congruent in claiming that the anomalies found are the probable cause of the crises. Structural etiologies include acquired causes such as stroke, trauma, and infections, or genetics such as many malformations of cortical development. In these cases, epilepsy is defined as "structural" as it is caused by the malformation, even in the presence of a genetic 
origin of the malformation. The identification of very small structural lesions requires appropriate magnetic resonance imaging studies that use specific protocols for epilepsy. Among the epilepsies of structural etiology, there are wellknown associations, such as hippocampal sclerosis associated with the mesial temporal lobe seizure. Other well-known associations are gelastic crises with hypothalamic hamartoma, Rasmussen's syndrome, and the picture of hemiconvulsionshemiplegia-epilepsy. The recognition of these associations is important to ensure that the patient's neuroimaging is carefully examined for a specific structural abnormality, also to consider epilepsy surgery if medical therapy is not effective. A structural anomaly can be supported by genetic or acquired causes, or both. For example, polymicrogyria may be due to mutations in genes such as GPR56, or be acquired following an intrauterine cytomegalovirus infection. The acquired structural causes include hypoxic-ischemic encephalopathy, trauma, infection, and stroke. Where a structural etiology has a welldefined genetic basis as in the case of tuberous sclerosis, which is caused by mutations in the TSC1 and TSC2 genes (which encode respectively for amartine and tuberin), both terms, "structural" and "genetic" can be used to define etiology.

b) Genetic etiology: A genetic etiology refers to a pathogenic variant (mutation) which has a significant effect in causing epilepsy. The epilepsies for which a genetic etiology has been admitted are varied and, in most cases, the underlying genes are not yet known. First of all, the hypothesis of a genetic etiology can be based exclusively on a family history of an autosomal dominant disease. For example, in Benign Neonatal Family Epilepsy syndrome, most families have mutations in one of the potassium channel genes, $\mathrm{KCNQ} 2$ or $\mathrm{KCNO}_{3}$. In contrast, in the autosomal dominant nocturnal epilepsy syndrome, the underlying mutation is currently known only in a small percentage of individuals. Secondly, a genetic etiology can be suggested by clinical research in populations with the same syndrome as Epilepsy with Childhood Absences or Juvenile Myoclonic Epilepsy. In terzis, a molecular basis may have been identified for the presence of mutations of single genes or the presence of a variant of the number of copies. The best-known example is Dravet syndrome in which over $80 \%$ of patients have a pathogenic variant of the SCN1A gene. In summary, to date, most genes have a heterogeneous phenotypic expression and, on the other hand, most syndromes are underpinned by genetic heterogeneity. When epilepsy follows a complex inheritance, which involves multiple genes and the presence or absence of an environmental contribution, susceptibility variants can be identified, that is, variants that contribute to the etiology but that are not sufficient in themselves to cause epilepsy. In scientific literature over 200 genetic mutations are known that are capable of causing or predisposing to epileptic seizures and most of these genes are directly or indirectly involved in ion channels. These include genes, ion channels, enzymes, GABAs, and $\mathrm{G}$ protein-coupled receptors. In homozygous twins, if one of them is affected, there is a $50 \%$ chance that the other will also be affected. In heterozygous twins, this risk drops to $15 \%$. However, these risks are greater in subjects with generalized seizures rather than with partial seizures and if both twins are affected, most of the time they have the same form of epileptic syndrome (70-90\%). Other close relatives of a person with epilepsy present a five times higher risk than that of the general population. Between $1 \%$ and $10 \%$ of those with Down syndrome and $90 \%$ of individuals with Angelman syndrome also suffer from epilepsy $[9,10]$.

c) Infectious etiology: The term infectious etiology refers to a patient with epilepsy, rather than a patient with seizures that occur in the context of acute infection such as meningitis or encephalitis. Common examples in specific regions of the world include neurocysticercosis, tuberculosis, HIV, cerebral malaria, subacute sclerosing panencephalitis, cerebral toxoplasmosis, and congenital infections such as the Zika virus and cytomegalovirus. An infectious etiology has specific implications for treatment. An infectious etiology can also refer to the development of epilepsy following an infection, as is the case with crises following the acute phase of a viral encephalitis.

d) Metabolic etiology: Several metabolic disorders are associated with epilepsy. The concept of metabolic epilepsy is that of a disorder in which seizures are the main symptom, which directly follows from a known or presumed metabolic disorder. The metabolic causes refer to a well-defined metabolic defect with systemic biochemical manifestations or alterations such as porphyria, uremia, amino acids, and pyridoxinedependent crises. In many cases, metabolic disorders have a genetic cause. Most metabolic epilepsies are likely to have a genetic basis, but some can be acquired such as brain folate deficiency.

e) Autoimmune etiology: The concept of an autoimmune epilepsy is that of a situation in which seizures are the main symptom, which results directly from an immune disorder Examples include anti-NMDA (N-methyl-D-aspartate) encephalitis and anti-LGI1 encephalitis.

f) Unknown etiology: Unknown etiology means that the cause of epilepsy is not yet known. Many epilepsy patients remain for whom the cause is unknown. In this category, it is not possible to make a specific diagnosis, which goes beyond the basic electroclinical semiology such as the epilepsy of the frontal lobe.

More or less known causes are certainly head injury, brain infections, dementias, compression syndrome, systemic vascular diseases, metal poisoning, cerebral hemorrhages, neurocysticercosis, multiple sclerosis, cerebral ischemias, 1 prolonged use of drugs, brain tumor, gliosis of hippocampal structures and anomalies of the cerebral cortex (agiria lissencephaly, pachygyria, band or nodular heterotopia and schizencephaly) In particular, the epilepsy that results from encephalopathy is called "Epileptic encephalopathy" and is the condition in which epileptic activity in itself contributes to severe cognitive and behavioral deficits and a greater extent than could be expected from the basic pathology alone (eg cortical malformation). Global or selective deficits can worsen over time. These deficits can have a spectrum of severity, accompany all types of epilepsy, and occur at any age. The concept of epileptic encephalopathy can be applied to epilepsies

Citation: Perrotta G (2020) Epilepsy: From pediatric to adulthood. Definition, classifications, neurobiological profiles and clinical treatments. J Neurol Neurol Sci 
at all ages and should be used more widely and not only for severe childhood-onset epilepsies. Many epileptic syndromes associated with encephalopathy have a genetic etiology, such as West syndrome, where there is a marked genetic heterogeneity, and Epileptic Encephalopathy with continuous wave-points during sleep (POCS), for which some genes have been identified .32 Equally, these syndromes may have an acquired cause such as hypoxic-ischemic encephalopathy or stroke, or they may be associated with malformations of cortical development which in turn may have a genetic or acquired cause. The concept of epileptic encephalopathy can also be applied to single-gene disorders, such as encephalopathies associated with mutations in the CDKL5 and CHD2 genes. However, a single gene can cause epileptic encephalopathy in some individuals and selflimiting epilepsy in others; examples include mutations of the SCN1A, SCN2A, SLC2A1, KCNQ2, KCNA2 and CHD2 genes. In an epileptic encephalopathy, the abundant epileptiform activity interferes with development, with consequent slowing down or regression of cognitive development; sometimes psychiatric and behavioral disorders are also associated. Epileptiform activity can cause regression in an individual with normal development or with pre-existing developmental delay, resulting in arrest or regression. A key element of the concept is that improving epileptiform activity could potentially improve development. From a clinical point of view, this is a crucial aspect, often reported by families and doctors. In many of these severe genetic disorders, developmental disturbance is due to the direct effect of the genetic mutation, as well as that of frequent epileptic activity. There are several ways in which this can manifest itself. There may be a pre-existing developmental delay, which is complicated by stopping or regression when seizures appear or if prolonged seizures occur. In other cases, the developmental slowdown may occur in a child with normal development, in which developmental slowdown occurs before the onset of frequent epileptic activity at EEG. A wellknown example is the encephalopathy of Dravet syndrome, in which the slowdown or regression of development occurs between 1 and 2 years of age, at a time when epileptiform activity on EEG is typically not yet abundant. This suggests that both developmental deficit and epilepsy are secondary to the mutation of the sodium channel subunit gene (SCN1A) which is found in over $80 \%$ of cases. In a third group, epilepsy may die out relatively early in the history of the child, but the developmental consequences may remain severe as observed in some patients with KCNQ2 encephalopathy or STXBP1 encephalopathy. These observations, which apply to many of the genetic encephalopathies, suggest the need to extend where appropriate, the terminology and to include the term "development", to recognize that both aspects (genetic cause and epilepsy) can play a role in clinical presentation. These concepts are fundamental for understanding the pathological process for both families and doctors $[8,11]$.

What is certain, however, concerns the study of the epileptic attack, how it occurs and what it causes, as it is universally recognized that it is the result of the excessive and abnormal activity of the neurons of the cerebral cortex [12-14].

It is estimated, according to statistical studies, that about $1 \%$ [15], of the world population can suffer from epilepsy (even if other data speak of $3 \%)[16,17]$ and that about $7-10 \%$ can be genetically predisposed the onset, at least once in their life, of epileptic seizures [18]. Epilepsy can, in serious cases, lead to death [19].

\section{Neurophysiological aspects}

From a neurobiological point of view, the discussion is more complicated. The electrical activity of the brain is normally not synchronous and is regulated by various factors both within neurons and in the cellular environment (these factors include the type, number and distribution of ion channels, changes to receptors and changes gene expression, while factors external to the neuron are related to ion concentrations, synaptic plasticity and regulation of the release of neurotransmitters by glial cells $[20,21]$; the epileptic seizure is, therefore, a paroxysmal (violent and exasperated) event through which epilepsy occurs, caused by the sudden, excessive and rapid discharge of a more or less extensive population of neurons that are part of the gray substance of the brain, according to the definition of John Hughlings Jackson). The aggregate of neurons affected by the discharge is thus defined as an "epileptogenic outbreak" [6].

The exact mechanism underlying epilepsy is not known in itself, but the pathophysiology at the cellular level is well known, however, it has not yet been established in what circumstances in the brain an excessive synchronization of neuronal activity occurs which then leads to epileptic attack. In cases of epilepsy, the resistance of the excitatory neurons to stimuli appears diminished during the period of a seizure. This can occur due to changes in ion channels or the improper functioning of inhibitory neurons. This then translates into a specific area from which epileptic seizures can develop. A further mechanism leading to epilepsy may be due to the "up" regulation of the excitatory neuronal circuits or the "down" regulation of the inhibitory circuits, following damage to the brain. These secondary epilepsies occur through processes known as "epileptogenesis". Impairment of the blood-brain barrier can also be a causative mechanism, as it would allow substances in the blood to enter the brain. There is evidence that epileptic seizures are not usually random events but are often caused by factors such as lack of sleep, stress, flashing lights, or sudden noises. "Epileptogenic threshold" is the term used to indicate the amount of stimulus needed for an attack to occur. In epileptic patients, this threshold appears much lower than in the healthy population. In epileptic seizures, a group of neurons begin to function abnormally suddenly, and synchronously. This causes a wave of depolarization, known as a paroxysmal depolarizing shift. Normally, after a neuron has had an electrical discharge, it becomes more resistant to other electrical discharges for some time. This is due in part to the effect of inhibitory neurons, to the electrical changes themselves within the neuron, and the negative effects of adenosine. Partial seizures originate in only one hemisphere of the brain, while generalized seizures begin in both. Some types of seizures can modify the structure of the brain, while others seem to have a lesser effect. Gliosis, neuronal loss, and atrophy of specific areas of the brain are related to epilepsy, but it is not clear if it causes these conditions or if they are the cause [22-26].

Citation: Perrotta G (2020) Epilepsy: From pediatric to adulthood. Definition, classifications, neurobiological profiles and clinical treatments. J Neurol Neurol Sci 


\section{Epilepsy in adults}

Generally, epileptic forms are classified according to strict technical schemes, based on the symptoms and the results of clinical investigations. In adults [27].

"Seizure epileptic seizure": It is the most common form and represents about $60 \%$ of the total epileptic episodes:

a) About $30 \%$ are "generalized" or "big bad" crises, that is, which originate from both hemispheres of the brain and can be "tonic convulsive crises" (motor manifestations with muscle hypertonus) and "clonic convulsive crises" (phases muscle contraction-relaxation cycles) or "mixed seizures";

b) The remaining $70 \%$, on the other hand, start with a "simple partial seizure" (ie involving a small brain region and there is no loss of consciousness) or "complex partial seizure" (evolution of the simple partial seizure, capable of involve multiple brain regions of the same hemisphere, with associated loss of consciousness). Partial seizures (also called "focal") therefore only affect a cerebral hemisphere but can nevertheless develop into generalized epileptic seizures, tending to spread.

"Non-convulsive seizure attack": The remaining $40 \%$ of overall seizures are of this type. It is the "typical absence" or the "little evil", which presents itself as a decrease in the level of consciousness, with a duration of about twenty to thirty seconds.

By schematizing as much as possible, synthetically and linearly, we distinguish the forms of seizure in adulthood $[6,28$ 33].

Typical absences: They are clinically characterized by a brief and isolated alteration of consciousness, with sudden onset and end. The subject abruptly interrupts the activity in progress, has a fixed gaze, if called generally does not answer; after a period ranging from 5 to 40 seconds, it resumes its activity as if nothing had happened. This central nucleus can be accompanied by minor motor phenomena such as small perioral and/or ocular clones (myoclonic absences), postural tone reduction (atonic absences), gestural automatisms, or motor perseveration (absences with automatisms); sometimes by vegetative signs such as initial loss of urine. All these clinical variants are accompanied by a typical critical EEG consisting of a bilateral, synchronous and symmetrical discharge of tipwave complexes at a frequency of $3 \mathrm{c} / \mathrm{s}$. Absences usually begin in the child and adolescent and regress between 15 and 20 years, but the adult may persist.

1) Atypical absences: Longer lasting and frequently accompanied by tonic, atonic or myoclonic motor components, often asymmetric, they show to the critical EEG anomalies similar to typical absences, but at a lower frequency (2-2.5 c ( s) and associated with rapid activities. This type of seizure is characteristic of epileptic syndromes with an unfavorable prognosis (for example, epileptic encephalopathies) with onset in childhood and generally persistent in adults.

2) Myoclonic seizure: They are characterized by the appearance of an involuntary, sudden and short ( $<100 \mathrm{msec})$ contraction of a muscle or group of muscles with variable topography (axial, proximal, distal), generally bilateral and symmetrical. A less frequent variant is the "negative myoclonus", consisting of a sudden interruption of muscle tone. Both positive and negative myoclonias are fleeting and are not accompanied by measurable alterations of consciousness. A very typical bilateral and synchronous discharge appears on the EEG consisting of several points followed by a slow wave (poly point-wave complexes). They are frequent in idiopathic generalized syndromes of the child, adolescent or young adult (juvenile myoclonic epilepsy); they hardly continue in the mature adult, where however they can be replaced by tonicclonic crises. They can be found in symptomatic generalized epilepsies (see below), where the prognosis is much darker.

3) Tonic-clonic seizure: They are the most known epileptic seizures in the collective imagination: known as "convulsions" and "the great evil", they are for many patients and the public "the seizures" par excellence. They are usually characterized by three main phases, tonic, clonic, and post-critical. The onset occurs with immediate loss of consciousness, tonic contraction of the whole musculature (duration 10-20 seconds), and fall; it is announced by a vocal emission caused by the violent contraction of the respiratory muscles. In this phase, lateral bite of the tongue, apnea with cyanosis of the face and important vegetative disorders (mydriasis, changes in blood pressure, heart rhythm disturbances, salivary hypersecretion, loss of urine, etc.) occur. The clonic phase follows (about 30 seconds) during which intermittent muscle decontractions, linked to the implementation of inhibitory circuits, generate bilateral and synchronous shocks which gradually decrease in frequency until they stop. The seizure ends, but the postcritical phase lasts many minutes or tens of minutes, in which the subject continues to present a disturbance of consciousness, is hypotonic, with a noisy breathing that slowly returns to the norm together with the restoration of contact with the environment. Often follows a long sleep. Diffuse muscle pain from broken muscle fibers, headache, and deep tiredness remains; relapsing shoulder dislocation is not uncommon. In laboratory tests, the increase in muscle enzymes creatinkinase and myoglobin is frequent. The critical EEG shows a rapid recruiting activity of low voltage, followed by sequences of tip and poly point-wave complexes that tend to gradually thin out in the clonic phase; a slow postcritical activity follows, which accompanies the gradual recovery of consciousness. The tonic-clonic seizure is a traumatizing event both for falls and for cardiovascular, respiratory, and muscular efforts. It can appear in all types of epilepsy, in idiopathic forms as a primary, elective, and sometimes unique manifestation, and then occurs preferentially upon awakening; in symptomatic forms as a secondary and conclusive manifestation of a focal seizure (a generalization of the seizure; see above). It is often facilitated by sleep loss, ethyl excesses and irregularities in taking therapy. The seizure may also be the epiphenomenon of non-epileptic morbid states, such as acute intoxications (for example ethyl or psychostimulant poisoning), encephalitis, meningitis. It appears in these cases as a generic neurological response to extreme conditions, in which an epileptogenic discharge, however, originated triggers the reticulocortical 
sequential paroxysmal activation and affects the entire cortex the structures of the brain stem that underlie wakefulness and muscle tone and culminates in a generalized muscle contraction and a coma. Neuronal and muscular energy consumption is very high, and combined with apnea it leaves a condition of neuronal exhaustion and general debilitation.

4) Clonic crises, tonic crises, falling spasms, atonic crises: They are fragments of the tonic-clonic seizure and paradoxically indicate in many cases a more serious neurological condition, in which plurifocal or diffuse damage to the brain seems to be an obstacle to the orderly reticulocortical sequence of the tonicclonic seizure. The so-called epileptogenic encephalopathies associated with deficient signs (psychomotor retardation, pyramidal signs, dystonia, etc.) appear in neurological pathologies of childhood. They are usually of difficult therapeutic control and often last, with various types, in adulthood. Clonic seizures are manifested by bilateral, rhythmic shocks, at a frequency of 2-3 c / s which symmetrically involve the same muscle districts, associated with a prolonged critical and postcritical disturbance of consciousness. The critical EEG shows bilateral discharges of tip and poly point-wave anomalies. Tonic seizures are characterized by a protracted muscle contraction associated with a brief alteration of consciousness. They can affect the muscles of the neck and head (axial tonic crises), the limbs (rhizomelic tonic crises) and also the limbs (generalized tonic crises), and easily cause violent and traumatic falls. The EEG shows rapid rhythmic and/or poly point points, with widespread expression. Flexion spasms are a tonic variant of the first year of life consisting, as the name implies, of a sudden flexion of the neck and trunk with abduction and flexion of the limbs, which appear in bursts and are the prelude to serious epilepsies of the child and adult. The EEG shows a disorganized path and a sudden desynchronization of the underlying activity at the spasm. The atonic crises are constituted by a sudden loss of postural tone that can only affect the neck with head fall, but generally involves the trunk and limbs and causes sudden and traumatic falls to the ground. The EEG shows rapid diffuse expression low voltage activity.

5) Focal seizure: Focal seizures (originally defined as partial) show an initial semiology that indicates the activation of a limited area of a cerebral hemisphere, the so-called epileptogenic zone. The first symptoms and signs, therefore, have a high localization value, and generally consist of a multiplicity of events, often in sequence, an expression of the anatomical-functional circuit affected by the seizure, its onset and during its propagation. The areas of onset and diffusion of the seizure change from patient to patient and in the different clinical conditions, but if the pathology is not evolutionary and does not compromise other areas over time, the symptomatological sequence presents a substantial monomorphism in the same patient, demonstrated with video recordings following. If the focal seizure does not affect the cortical structures that underlie consciousness, and the patient "attends" to his seizure and can describe it. If, on the other hand, consciousness is compromised, the patient "loses" many details of the seizure; moreover, the seizure exposes too much greater risks. The disturbance of conscience conditions, in addition to the accuracy in reporting critical symptoms, the ability to defend oneself from environmental dangers, and also to be aware of the very occurrence of seizure (a condition that evaluates therapies uncertain). It is therefore an aggravating factor, especially if consciousness is disturbed from the beginning. The classification of the seizure of 1981, inspired by Henry Gastaut and still practically in use, therefore appropriately distinguishes focal seizure into simple, with a conserved conscience, and complex, with disturbance of conscience.

6) Simple partial crises: Simple partial seizures occur with highly variable symptoms, depending on the cortical area where epileptogenic discharge develops. The seizures are therefore distinguished according to the clinical manifestations:

a) Motor crises: The crises of the primary motor area (rolandic cortex, from which the denomination of rolandic crises) can express themselves with clones limited to a single district (e.g. the hand) or highlight the progressive recruitment of contiguous cortical areas, for example, hand $>$ face $>$ language (seizure or Jacksonian march), progression of high localization value. If the premotor cortical areas are primarily involved, the manifestations show mainly tonic-postural characteristics. The motor signs can then be expressed in different ways depending on the interest of other structures responsible for controlling movement: in fact, versive crises (lateral deviation of the head and eyes) from the involvement of the lateral frontal cortex, phonatory crises from the involvement of the cortex can be observed opercular, motor crises with inhibitory signs (motor inhibition, language arrest, etc.) from affecting some frontal cortical areas (for example the supplementary motor area).

b) Some-sensitive seizure: If the seizure starts in the primary sensory area (posttrolandic cortex), the clinical manifestations are made up of elementary sensory symptoms (tingling, a sense of falling asleep, a feeling of electric shock, pain, etc.) localized to the contralateral hemisome. Also in this case a sensitive Jacksonian march may be present.

c) Visual crises: They are characterized by elementary lateralized hallucinatory manifestations (colored and moving luminous dots, flashes, flashes), structured (scenes, characters), or illusionary manifestations such as shrinking, enlargement and distortion of the visual field, called micropsia, macropsia, dysmorphia; negative symptoms such as scotomas, hemianopsia, etc. may also be present. In elementary hallucinations, the critical discharge involves the occipital primary visual area (area 16 and 17) contralateral, in other cases it affects the posterior and parieto-occipital temporal cortex.

d) Auditory seizure: Also, in this case, elementary hallucinations (noises, sounds), complex (voices, music), or illusionary manifestations such as distancing, amplification, or deformation of sounds appear. The localization of critical activity occurs in the primary auditory cortical areas (Heschl's laps, the posterior portion of the superior temporal gyrus) and surrounding areas. 
e) Dizzying crises: Rare and difficult to diagnose if clinically isolated, they manifest themselves as an impression of rotation of the external environment or displacement of the body concerning the external environment. The posterior temporal-occipital and inferior parietal temporal cortex is affected by the critical discharge.

f) Olfactory and gustatory crises: Also known as hook crises, they are characterized by olfactory and gustatory illusions/hallucinations usually unpleasant (burnt gum, tar, acid), often associated even if processed in different areas: in olfactory crises the basal frontal cortex (rectum and orbital cortex), in gustatory crises the parietal operculum.

g) Vegetative seizure: The vegetative signs are very frequent in crises and can present themselves as a symptom of onset, even if they often appear during the episodes. The manifestations can be multiple and affect the pupils (mydriasis), thermoregulation (horripilation, heat, sweating, chills), the cardiovascular system (pallor or redness of the face, heart rhythm disturbances, hypertension or rarely hypotension), the gastrointestinal system (abdominal sensations, hypersalivation, nausea, belching, vomiting). A strange feeling in the stomach that goes up towards the throat (known as "epigastric aura") is very frequent, one of the bestknown manifestations of epilepsy, which can be the symptom of the onset of crises in the medial part of the temporal lobe but can also accompany crises that begin with the island operculum or the prefrontal cortex. Rarely, a slowing of the heart occurs that causes blood pressure and syncope to drop.

h) Cognitive and affective crises: They are among the most specific epileptic manifestations. They concern memory, the flow of experience, emotions. The patient has the vivid impression of having already experienced or seen the present situation (dejà vu and dejà vecu), feels a sudden sensation of estrangement (jamais vu), of unreality (dream state), of detachment from one's person (depersonalization) or it, is pervaded by a memory that paralyzes the mind (hypermnesia, dismnesia); affective crises consist in the sudden emergence of an uncontrollable emotional state (anger, fear, joy). Automatic involuntary oro-alimentary activities (chewing, swallowing), gestures (scratching, rubbing, manipulating objects, etc.), mimics, verbal appear; the behavior is rarely aggressive. The lateral and medial temporal areas, the cingulate gyrus and the frontotemporal basal cortex are involved in all these events.

7) Complex partial crises: They are crises with impaired consciousness. The disturbance of conscience depends on the decommissioning of the associative cortical areas, by invasion by the critical discharge or by deactivation of the Default Mode Network. Being able to establish the level of consciousness confidently based on the anamnestic report is very difficult and requires very careful observation and testimony. It is verifiable based on the reactivity to environmental stimuli and the ability to memorize events, it is clinically manifested by stopping the activity in progress, gaze fixation, inadequate response, and loss of memory of simple questions and orders. When it is verified by an observer it is defined as breakage or loss of contact. It can rarely go unnoticed and be denied by the patient, and emerge in the video-EEG recordings integrated by the interaction with the doctor or technician. Despite the diagnostic difficulties, the proposal to neglect the consciousness disorder as a classification parameter does not agree with many clinicians, who are rarely considered satisfied if the therapy manages to prevent the seizure from ending with the loss of consciousness. Impairment of consciousness takes on greater clinical severity when it appears without warning at the beginning of the episode, since it does not allow for any defensive strategy. For this reason, it is appropriate to try to identify complex crises with loss of initial consciousness compared to those in which consciousness is compromised during the episode, after a focal phase with a clear conscience. In crises with conscience disturbance, the emergence of automatisms (hence the definition of psychomotor crises) consisting of verbal, gestural, and mimic activities produced by the invasion of motor structures by epileptogenic discharge (critical automatisms) or by depression of consciousness is frequent (release automatisms).

\section{Epileptic forms based on brain localization}

Still schematizing as much as possible, synthetically and linearly, we now distinguish the forms of epileptic syndromes based on brain localization [6,28-33].

1) Epilepsy of the temporal lobe: The temporal lobe is the ideal terrain for the development of epilepsy, and the one where most adult epileptic seizures are structured. The main reason is its anatomical construction, the susceptibility of some areas to the lack of oxygen, and the almost unique possibility for the brain to generate new neurons. Last but not least, the considerable rearrangement that the medial temporal structures undergo in fetal development and the consequent greater frequency of malformative pathologies. The temporal lobe preserves the most archaic structures, those of memory (the fundamental building block to elaborate an individual story) and those of smell (the first sensory approach to the knowledge of the environment), structures rolled upon themselves (such as the hippocampus) and parallel circuits (such as olfactory areas). The hippocampus is a vascular border area of the terminal vessels of the posterior cerebral, and like all border areas, it is more sensitive to ischemia. It also has one of the few areas (the subgranular zone of the dentate gyrus) where new neurons are generated in adulthood. All these are ideal conditions for building circuits that feed themselves by structuring repetitive discharges. The temporal lobe is divided into two areas that reflect two evolutionary times: the medial, archaic portion, which contains the entorhinal and para-hippocampal cortex, the dentate gyrus and the actual hippocampus, consisting of three-layer bark (allogeneic) phylogenetically ancient, which underlies the functions of smell, memory, and emotions and regulates vegetative functions; and a lateral portion, which includes the three lower, middle and upper convolutions, consisting of phylogenetically more recent six-layer bark (neocortex), which underlies the functions of hearing and language. It is evident from what has been said that it is the medial portion, which contains the hippocampus, the one favored by epileptic discharges. The hippocampus can be called "the magic ring of epilepsy". There are therefore two major 
categories of temporal epilepsy, the medial and the lateral with many exchanges between them due to the territorial contiguity:

a) Medial temporal epilepsy: The most frequent pathology, and by far the most common cause of epilepsy, is sclerosis of the hippocampus. It is formed following even brief phases of cerebral anoxia, an event at risk at birth, in the transition from placental to pulmonary circulation, when a delay in the expulsion phase of the fetus can interrupt the oxygenation of the blood for several minutes, and the hippocampus it is the first structure to suffer. A second condition is the febrile seizures of the first three years, in which the convulsive discharges favor the hippocampus and neighboring areas and where the energy consumption causes damage from hyperactivity (called glutamatergic due to the glutamate mediator involved). Loss of many neurons occurs and, in the following months and years, budding of new fibers, the genesis of new neurons, and the formation of synapses and new recurrent circuits are activated. Other etiologies are benign tumors (astrocytomas, gangliocytomas, neuroepithelial dysembryoplastic tumors, known with the English acronym DNET), vascular malformations (cavernous angiomas) or cortex (focal dysplasias). The clinical manifestations are characterized by vegetative signs: the most common is the epigastric aura, an annoying sensation of pressure that rises from the stomach region towards the throat (the most frequent epileptic symptom ever); still salivation, nausea, pallor. Also, experiential signs (already seen, already lived, never seen, extraneousness, dreamy state, depersonalization), psychic (fear, anxiety, anger), sensory (strange flavors and smells). Consciousness is generally compromised and oroalimentary (sucking, chewing, swallowing), gestural (mimic expressions, manipulations of objects), verbal (sounds or words), rarely outpatient, emerges. The most typical crisis evolves with epigastric aura, fear ("I am afraid that the seizure will come to me", reports the patient), confusion, chewing (movements of the jaw as with a candy in the mouth, which French epileptologists call "machonnement"). The prognosis is not good, since about one in three patients does not respond to medical treatments and becomes a possible candidate for surgical resection of the epileptogenic area. The early appearance of verbal automatisms is very important clinically and should always be investigated, as it denotes the rapid invasion of the centers of speech and makes surgical therapy more at risk.

b) Lateral temporal epilepsy: The causes are the same as for the medial temporal form. Hippocampal sclerosis does not constitute the main etiology, as in the medial form, but is still present since in some cases tissue sclerosis also involves lateral structures from where the clinical seizure starts. The manifestations are acoustic, dizzying, experiential, and cognitive sensory; moreover, in some cases, the seizure starts with epigastric sensations dating back. The motor manifestations have more elementary characters and alongside the automatisms, there are dystonic postures of the upper limb, clonic movements of the contralateral limbs, later version of the trunk; Verbal automatisms are frequent in the outbreaks of the dominant hemisphere. Consciousness is compromised. The prognosis does not differ from the medial form.
2) Epilepsy of the frontal lobe: The frontal lobe is the most recent phylogenetically structure, developed especially in primates and humans. It preserves, in the anterior rolandic cortex (the convolution placed in front of Rolando's sulcus) and in the immediately anterior areas, the first and secondorder elementary motor structures, and in the basal portion phylogenetically ancient areas with an olfactory function and for regulating emotions. Typical of man are the structures of production of the language, developed in the lateral part and lateralized on the left for the propositive components and on the right for the emotional components of the language as well as the structures of the anterior portion (prefrontal cortex) that control the behavior and direct actions for one purpose, in their cognitive components with the dorsolateral portion and the emotional components with the ventromedial portion. Front-end crises are much less frequent than temporal crises, but have numerous possibilities of expression. The crises of the primary motor area are manifested by contralateral focal clones, which can progressively propagate along the cortex involving fingers, hand, lips, upper limb, and the entire contralateral hemicbody (Jacksonian seizure, by the English neurologist Huglings Jackson who has them described). The involvement of the front-mesial structures (premotor area, in particular the additional motor, and the gyrus of the cingulum) lead to much more complex, bilateral, asymmetrical movements, with the assumption of abnormal postures and, sometimes, with automatisms of the limbs comparable to the movements swimming, cycling and kicking. They are accompanied by exaggerated mimic expressions and primitive vocalizations (grunts, rumblings, scraping of the throat). Automatisms are different from case to case but always the same in the individual patient. These crises, the most typical of frontal outbreaks, are called "hyper motor crises", are often nocturnal, short, with abrupt and frequent beginnings and ends, with a consciousness usually preserved and without postcritical confusion; with cluster trends. They are therefore very similar to the autosomal dominant idiopathic frontal epilepsy seizure. Carbamazepine is the drug of choice, but the response to therapy is uncertain. Since the additional motor cortex is an area with sensorimotor integration functions, curious sensations may appear such as pressure on the head and trunk, lightness, levitation, dizziness. The involvement of the areas of language manifests itself with elementary vocalizations or with the arrest of language. Consciousness is usually spared. Forces of the anterior cortex appear "forced thinking" (ie an idea or mental image that imposes itself with irresistible force and sudden suspensions of consciousness similar to absences, with contralateral deviation of the eyes and head) and other cognitive alterations (depersonalization, etc.). Olfactory and gustatory sensations, vegetative signs, and sometimes even epigastric sensations, automatisms may appear in the frontonasal origins.

3) Epilepsy of the parietal lobe: The parietal lobe has elementary sensitive functions located in the cortex behind the central furrow of Rolando (post-rolandic cortex) and concerning the somatic sensations of the opposite half of the body (touch, position, and movement of the limbs, vibration, heat and cold, pain). The sensory area is delimited posteriorly 
by the intraparietal sulcus that separates it from the posterior portion of the lobe, formed by associative cortex: this area, which continues indistinctly with the temporal and occipital associative cortex, and the area richest in neurological functions "Superior" of the entire cortical mantle (organization of space, recognition of the shapes of objects, awareness of one's own body, selective attention, ability to calculate and plan motor strategies on the left and decipher the grammatical and syntactic components of language). The most frequent crises are those of the somatosensory area and consist of tingling sensations, falling asleep, crawling, constriction, heat, cold and sometimes pain localized in the opposite half of the body, with greater frequency on the face, thumb and fingers of the hand, that is, on the areas that have the most extensive representation in the bark. The sensation can move along the body in a march similar to the motor one (jacksonian sensitive seizure). Sensations of modification of the position and movement of the limbs, or dislocation of the body or vertigo appear more rarely. Sometimes blockage of understanding of language or distortion of vision with moving away or closer to objects. Since these are subjective events, the conservation of consciousness and accurate reports are necessary, with the risk of exchanging epileptic events with psychogenic manifestations. Etiology and therapy do not differ from other focal crises; the area is one of the most frequent sites of arteriovenous angiomas.

4) Epilepsy of the occipital lobe: It includes the primary and secondary visual areas, located in the medial portion of the occipital pole and the surrounding areas of the lateral occipital cortex, and areas with an associative function that continue with the temporoparietal associative cortex and which are left to decipher the written language and visual symbols (for example road signs), on the right to the spatial orientation and bilaterally to the recognition of objects and faces, to the visual orientation and the organization of eye movements. The most frequent crises consist of elementary visual hallucinations, consisting of small dots, stripes or colored spirals that move with circular motion occupying an area of the contralateral visual field, for a duration of tens of seconds or one to two minutes. Conjugated eye movements can be associated. Consciousness is preserved. If the circuit involved affects the portion of the border with the parietotemporal association areas, complex hallucinations regarding scenes or objects may appear. Sometimes the seizure causes blurred vision. Headache may follow more frequently than in other lobe seizures. Visual crises are differentiated from migraine auras, which are characterized by intermittent zigzag, non-colored visual hallucinations, which move and spread like wildfire in the visual field starting from a peripheral area but do not have spiral motion, they leave a blind area and last from 10 to 20 minutes. Etiology does not differ from other lobes; characteristic etiologies, although rare are calcifications secondary to celiac disease, encephalo-trigeminal angiomatosis (Sturge-Weber disease), the results of eclampsia.

5) Hypothalamic hamartoma epilepsy: The hypothalamus is an area located in the center of the brain, around the third ventricle responsible for autonomic and endocrine regulation functions. It develops an exclusive and characteristic form of epilepsy that appears in the hamartomas (malformative tumors consisting of neuronal and glial tissue) of this area and has as its main characteristic an unmotivated and sudden laugh seizure (the so-called gelastic seizure) or, even if very rarely, a seizure of sudden crying (diacritic seizure). This epilepsy can sometimes have a catastrophic evolution, with the appearance of a fall seizure or an evolution towards an encephalopathy.

6) Other syndromic entities: A particular form of frontal epilepsy affecting the rolandic regions is Kojewnicow syndrome, also called continuous Epilepsia partialis, characterized by sub-continuous clones affecting the contralateral hemisome, expression of motor focal evil state. The form is often secondary to malformative lesions (cortical dysplasias). Another nosographic entity that can be assimilated from a clinical point of view to the previous one is Rasmussen encephalitis (or syndrome), an immune-mediated pathology with the presence in the blood of antibodies directed towards the glutamate receptor (antibodies antiGlu3). It is characterized by the association of continuous partial epilepsy, hemiparesis, cognitive disorders, and resonance finding of progressive cerebral hemiatrophy. More frequent in childhood, in addition to antiepileptic drugs, it requires immunomodulating treatment (plasmapheresis, IV Ig, steroids) and, in resistant and rapidly progressing cases, surgical therapy (often hemispherectomy).

\section{The seizure seizure and the difference with the epileptic seizure}

From the classic "epileptic seizure ", however, it is necessary to distinguish the so-called "convulsive seizure": with the latter term we want to indicate violent and involuntary contractions affecting some voluntary skeletal muscles (without loss of consciousness) and are caused by other conditions: abscesses brain; withdrawal seizure (alcohol withdrawal, drugs, drugs); encephalitis; fever (we speak of febrile seizures, especially in children); meningitis; brain tumors; metabolic problems (hypoglycemia, hyponatremia and hypoxia); tetanus infection; hyperuricemia. About 5-8\% of epileptics experience seizures that are often triggered by specific events; this condition is known as reflex epilepsy. Those who suffer from this particular disorder have seizures that are activated only by specific stimuli. The most common triggers can be flashing lights and sudden noises. In some types of epilepsy, seizures occur more frequently during sleep and sometimes almost exclusively [29,34-36].

\section{The non-epileptic seizure}

Finally, we can also speak of "non-epileptic seizures" if caused by external agents such as electroconvulsive therapy (TEC, the common "electroshock"), or convulsive drugs. Sometimes it can be even difficult to differentiate them from syncope, hysterical seizure, and simulation or from other pathologies that are actually at the origin of the epileptic seizure, as in the case of Bourneville tuberous sclerosis, West Syndrome, Lennox Syndrome- Gastaut, Down syndrome or Landau-Kleffner syndrome [29].

\section{The recent revision of the classification of epilepsies}

A recent worldwide classification review has introduced a new way to study them. From a strictly clinical point of view,

Citation: Perrotta G (2020) Epilepsy: From pediatric to adulthood. Definition, classifications, neurobiological profiles and clinical treatments. J Neurol Neurol Sci 
the known and classified epileptic forms always generate a passionate debate in the academic field because it is built on the combination of the complex concepts that underlie the diagnosis of epilepsy, in part because it represents a crucial tool for our daily practice. The classification is based on the opinions of expert epileptologists and experts in related disciplines from all over the world. There is no doubt that the objective must be a classification based on scientific evidence, however, at present, our knowledge is not yet sufficient to build a classification based on rigorous scientific bases. The current proposals are therefore based on the combination of the latest scientific knowledge, with the opinions of high-level experts, worldwide. The new "Classification of Epilepsies" is structured on three multiple levels, to allow the classification of epilepsy in different clinical situations. The great variability of resources available in the world requires a classification on different levels to allow the clinician to make the diagnosis based on the available resources. Where possible, a diagnosis should be made on all three levels and the etiology identified [8].

The starting point of the classification structure (first level of diagnosis) is the "Type of seizure"; it is assumed that the clinician has already made a definitive diagnosis of epileptic seizures, therefore a diagnostic algorithm is not considered to distinguish epileptic from non-epileptic events. The classification of the type of seizure is following the new nomenclature of the accompanying document. Based on the onset characteristics, the seizures are classified into focal, generalized, and unknown onset. In some situations, in the absence of EEG, video, and image studies, the classification based on the type of seizure is the highest possible level for diagnosis. In other cases, the information may not be sufficient for a diagnosis of a higher level, as happens for example when a patient had a single seizure [8].

The second level of diagnosis is that of the "Type of Epilepsy", and assumes that the patient has a diagnosis of epilepsy based on the 2014 definition. In addition to the well-defined "Generalized Epilepsies" and "Focal Epilepsies", two new ones are inserted categories: "Generalized and Focal Combined Epilepsy" and "Unknown Epilepsy". Many epilepsies include different types of seizures; in case of diagnosis of Generalized Epilepsy, the patient's EEG typically shows generalized tipwave anomalies. People with generalized epilepsies can have various types of seizures, including absences, myoclonic seizures, atonic seizures, tonic seizures, and tonic-clonic seizures. The diagnosis of generalized epilepsy is based on clinical characteristics, supported by the presence of typical intercritical discharges to the EEG. In the case of a patient with generalized tonic-clonic seizures and a normal EEG, classification requires caution. In this case, further evidence is needed for diagnosis such as the presence of myoclonic seizures or a significant family history. Focal epilepsies include focal or multifocal crises, as well as crises affecting a hemisphere. Various types of focal crises can be recognized, including focal crises with or without compromised contact, focal motor and non-motor crises, and focal crises that develop into bilateral tonic-clonic crises. Intercritical EEG typically shows foca epileptiform abnormalities, but the diagnosis must be based on clinical criteria and supported by EEG results. There is a new group of epilepsies, defined as "Generalized and Focal Combined Epilepsies", since some patients present with both generalized and focal seizures. Again, the diagnosis is based on clinical features, supported by EEG findings. EEG seizure documentation is useful but not essential. Intercritical EEG can show both focal epileptiform abnormalities and generalized anomalies, however, epileptiform activity is not essential for making the diagnosis. Dravet syndrome and LennoxGastaut syndrome are two common examples of conditions in which both types of seizures occur. Diagnosis of the type of epilepsy can also be the highest diagnostic level reached by the clinician when it is not possible to diagnose a specific epileptic syndrome. Here are some examples: the situation of a child or adult with non-lesional temporal lobe epilepsy is frequent, in this case, the diagnosis is of focal epilepsy of unknown etiology. Another example is that of a 5-year-old boy who has generalized tonic-clonic seizures and generalized wave anomalies generalized to EEG: in this case, the diagnosis is certainly that of Generalized Epilepsy, even if classification in a well-defined syndrome is not possible defined. A third example is the less common scenario of a 20-year-old woman who presents both focal seizures with altered contact and absence seizures, with epileptiform anomalies both focal and generalized to EEG and normal Magnetic Resonance: in this case, the diagnosis is from Generalized and Focal Combined Epilepsy. The term "Unknown Epilepsy" is used to describe patients who have Epilepsy but the clinician is unable to define whether the Epilepsy Type is focal or generalized due to lack of sufficient information. This could be due to different reasons: EEG not available or non-informative EEG (for example because normal). If the Type-Types of Seizure are unknown, then the Type of Epilepsy may also be unknown for similar reasons, although the type of seizure and type of epilepsy may not always be in agreement. For example, the patient may have had several symmetric tonic-clonic seizures without normal focal and EEG characteristics. So the onset of the seizures is unknown and the patient has epilepsy of an unknown type [8]

The third level is the diagnosis of "Epileptic Syndrome". An epileptic syndrome is defined by the association of specific characteristics that include types of seizures and findings from the EEG and neuroimaging. Syndromes often have age-dependent characteristics: age of onset and possibly remission, triggers of crises, circadian variations, and sometimes prognosis. It can also be characterized by specific comorbidities, such as the presence of an intellectual disability and psychiatric disorders, as well as peculiar EEG and neuroimaging characteristics. The definition of a syndrome can also have etiological, prognostic and treatment implications. It is important to note that there is not necessarily an unambiguous correlation between an epileptic syndrome and an etiological diagnosis and that the definition of an epileptic syndrome can be useful for improving patient management at different levels. There are many well-recognized syndromes, such as Epilepsy with Childhood Absences, West syndrome, and Dravet syndrome [8]. 
Lastly, the "state of epileptic illness" deserves a separate mention: the epileptic seizure usually lasts no more than two to three minutes; when this does not happen and is prolonged considerably or repeated cyclically, we will speak of a "state of epileptic evil", and also here we will speak of partial forms or generalized forms. It is a condition capable of creating serious brain injuries to the patient, until death [6].

\section{Epilepsy in children and adolescent}

However, epileptic syndromes can also arise and occur in children and adolescents. Let's see them in detail [37]:

\section{Epileptic syndromes with onset in the neonatal era}

a) Benign familial neonatal crises (BFNSs): Rare syndrome with typical onset at 2-3 days of life. Normal psychomotor development. The seizures are short-lived (1-2 minutes) and can be very frequent (up to 20-30 per day). Many crises begin with tonic motor activity with apnea, followed by vocalizations, eye movements, autonomic signs, motor automatisms, and focal or generalized clones; the epileptic state does not occur. The seizures resolve spontaneously 1 to 6 months after the onset. About $10-14 \%$ of patients will develop other types of seizures in future years. Positive familiarity for similar episodes in the neonatal era; Linkage studies have shown mutations on chromosomes $20 \mathrm{q}$ or $8 \mathrm{q}$ at the level of genes coding for subunits of voltage-gated potassium channels.

b) Benign neonatal crises (SNBs): Sporadic cases described. Short-term clonic crises that become progressively more frequent, associated with apnea crises and sometimes epileptic state. The intercritical child is normal. The age of onset is typically between 4 and 6 days of life (in the past defined as "fifth-day seizure "). The prognosis for the risk of recurrence of other crises in the future and psychomotor development is good.

c) Early childhood onset epileptic encephalopathy (IEEE or Ohtahara syndrome): Generally onset in the first 10 days of life. The typical seizure is represented by a tonic spasmlike movement of longer duration than that typical of West syndrome. These seizures can occur in isolation or clusters with the duration of each tonic seizure from 1 to 10 seconds and a frequency of these episodes ranging from 10 to 300 in 24 hours. Crises can be generalized and symmetrical or lateralized; focal clonic motor crises with erratic features can occur less frequently. Most often there is a serious structural anomaly of brain development. The EEG has a "suppressionburst" pattern. The negative prognosis for psychomotor development and increased risk of mortality. A future evolution in West syndrome or Lennox-Gastaut syndrome is possible for surviving children. Pharmacoresistant epilepsy.

d) Earlymyoclonic encephalopathy (EME): Debut in the first days of life. EEG pattern "suppression burst". It is distinguished from Ohtahara syndrome by the presence of an intense highfrequency myoclonus with migrant characteristics; Focal clonic seizures or tonic seizures can occur. Often associated with metabolic diseases (e.g. non-ketotic hyperglycinemia). e) Epilepsy with focal migrant crises: Condition with features not yet well-defined onset in the first weeks of life. Rapidly worsening epilepsy with focal or multifocal crises characterized by variable ("migrant") localization of the prevalent outbreak. Pharmacoresistant form with a negative prognosis.

\section{Childhood-onset epileptic syndromes}

a) Infantile spasms (ISs or West syndrome): Age of onset generally between 4 and 6 months (more rarely they can occur in the late neonatal period or after 12 months). The crises are represented by the typical spasms in clusters that occur mainly in waking; spasms can be in flexion or extension; a focal brain alteration can cause asymmetric spasms. The period of spasms is associated with a psychomotor regression with a reduction in visual attention and interaction and an increase in irritability. Depending on the etiology, "symptomatic" (about $90 \%$ ) or "presumed symptomatic" forms are distinguished; genetically determined forms have been described (CDKL5 gene in females and ARX gene in males). West syndrome is defined by the combination of spasms with a very characteristic EEG pattern, called "hypsarithmia". The most effective therapies are represented by the administration of adrenocorticotropic hormone (ACTH), or by high doses of oral corticosteroids or, in the case of symptomatic infantile spasms of tuberous sclerosis, by vigabatrin.

b) Benign myoclonic epilepsy of childhood (BMEI): Rare condition (represents about $1 \%$ of idiopathic generalized epilepsies). Debut between 4 months and 3 years of life. Myoclonic seizures mainly involve the head, eyes, upper limbs, diaphragm, and more rarely the lower limbs (in this case they can cause occasional falls); myoclonic seizures can occur in isolation or short clusters. Normal psychomotor development. Male $/$ female ratio $=2: 1$. Normal intercritical EEG; myoclonic seizures have an EEG correlation of tip-wave or poly pointwave discharges. The drugs used for treatment are valproic acid or other broad-spectrum antiepileptics.

c) Severe myoclonic epilepsy of childhood (SMEI or Dravet syndrome): It probably represents $1-3 \%$ of epilepsies with onset in the first year of life; typically onset between 5 and 12 months with recurrent episodes of epileptic state during fever (often focal / lateralized seizures). The types of seizures that can occur are clonic seizures associated with fever, myoclonic seizures, atypical absences, and complex focal seizures. Before the onset, psychomotor development is normal; from the second year of life, different types of crises occur with the prevalent myoclonic component (especially starting from 18 months). Heat (fever or even a hot bath) is a trigger for crises. The intercritical EEG shows generalized, focal or multifocal anomalies, and can detect photosensitivity. Family history of epilepsy and / or febrile seizures in $15-25 \%$; in about $70 \%$ of cases there is a mutation in the SCN1A gene. The most effective drugs are valproic acid and clobazam in association with stiripentol; carbamazepine and lamotrigine worsen critical symptoms. A clear regression of psychomotor development typically occurs about a year after the onset of the seizure. Increased risk of sudden death (Sudden Unexpected Death in Epilepsy or SUDEP). 


\section{Epileptic syndromes with onset in pre-school age}

a) Epilepsy with myoclonus-astatic seizure (EMAS or Doose syndrome): Rare condition, slightly more common in men and positive familiarity in about a third of cases. Debut at the age of 2-5 with frequent fall crises; falls can be caused by myoclonusastatic crises and / or atonic crises. Myoclonus-astatic crises, characteristic of this condition, can be associated with atonic, myoclonic crises, and absence crises; myoclonus-astatic epileptic status is common. The EEG can be normal in the early stages and later characterized by slowing down at biparietal level, generalized tip-wave discharges slow, and irregular tipwave discharges associated with myoclonus-astatic crises. Valproic acid is the most effective drug as it acts against myoclonic crises, atonic crises, and "absences"; in cases with resistant crises, low-dose lamotrigine in combination with valproic acid can be effective; other drugs used in treatmentresistant forms are ethosuximide and benzodiazepines. The prognosis is variable.

b) Progressive myoclonic epilepsies: This category includes several forms of epilepsy (e.g. myoclonic epilepsy with ragged-red fibers or MERRF, Lafora disease, UnverrichtLundborg disease, etc.) which may initially have characteristics similar to those present in Doose syndrome, but which later they differ from this form due to the presence of relevant neurological anomalies and to the delay/deterioration of cognitive development. The triad present in these patients is represented by different types of seizures (including myoclonic seizures), the presence of neurological signs, and progressive deterioration.

\section{Epileptic syndromes of the child}

a) Epilepsy absences of the child (CAE): Also defined with the term "pycnolaxia" (critical episodes that tend to occur very frequently, from tens to hundreds of times a day); the seizure occur with sudden and brief loss of contact with the surrounding environment, associated with a lack of response to external stimuli and the interruption of voluntary activities in progress (duration of episodes of "absence" generally from 4 to 20 seconds); peri-oral and peri-ocular districts and / or automatisms may be present; seizures are typically triggered by hyperventilation (a maneuver, which can be used in the clinic and which can evoke a crisis of "absence" in more than $90 \%$ of children with EWC, consists in allowing the patient to breathe deeply for about 3 minutes making him keep his arms stretched forward and inviting him to count his breathing). A standard EEG in typical forms is sufficient for diagnosis. Age of onset between 4 and 10 years (peak age between 5 and 7 years; more frequent in girls). Strong genetic component with positive familiarity in one-third of cases and risk of recurrence in children of about $10 \%$. Generalized tonic-clonic seizures can occur, albeit rarely, generally long after the onset of "absences" (usually in adolescence after the remission of "absences"). The intercritical EEG is normal; absence seizures have a correlated EEG represented by generalized peak-wave discharges and wide voltage at about $3 \mathrm{~Hz}$. The first choice antiepileptic drugs are ethosuximide (it does not protect against any generalized tonic-clonic crises), acid valproic, and lamotrigine; carbamazepine treatment is contraindicated as it can aggravate epilepsy. The prognosis for the disappearance of absence crises is very good (disappearance of crises in general before the age of 12); there is an increased risk in adulthood of developing generalized tonic-clonic seizures.

b) Epilepsy with Myoclonic Absences (EMA): Age of onset between 2 and 13 years. Male prevalence. $50 \%$ of patients have normal psychomotor/cognitive development at the onset. Crises are typically characterized by abrupt "absences" with marked rhythmic and synchronous myoclonus which symmetrically affects the limbs; mouth, chin, eyes, and eyelids may be involved; seizures typically last less than a minute; in a third of cases, generalized tonic-clonic crises, pure "absences" and astatic crises can occur. There is a familiarity for epilepsy in $25 \%$ of cases; many cases remain of unknown etiology. Negative prognosis for cognitive development and seizure control. The most effective treatment is generally represented by the combination of valproic acid with ethosuximide or lamotrigine.

c) Epilepsy absences with eyelid myoclonus (Jeavon syndrome): Onset between 2 and 14 years with peak at 6-8 years; most frequently found in women. Short crises (lasting about 3-6 seconds), spontaneous or precipitated by closing the eyes in an illuminated environment (not in the dark); crises characterized by a deviation of the gaze upwards and retropulsion of the head with eyelids that present repetitive clones with the possible association of the compromised state of consciousness. The EEG detects short generalized tip/ poly point-wave discharges, of wide voltage at 3-6 $\mathrm{Hz}$ and photosensitivity.

d) Benign epilepsy with central-temporal points (BECTS or Epilepsy rolandic): It represents the most common focal epilepsy of the child; the age of onset between 3 and 13 years; more common in males. Focal crises involving the facial/perioral district, which can evolve with a secondary generalization; seizures occur in $80 \%$ of cases in the sleep phase; typical features include a one-sided feeling of numbness/paraesthesia at the level of the tongue, gums or cheeks, guttural sounds or speech arrest, hypersalivation, difficulty swallowing or post-critical sialorrhea, involuntary movements or tonic contractures of the tongue or jaw, clones interesting part of the face. Intercritical EEG is characterized by the presence of slow phasic points at the level of the central-temporal regions, which can have onesided localization and which increase in sleep frequency. If the clinical and EEG characteristics are not typical, it is advisable to carry out a brain MRI examination to exclude a form of lesional epilepsy. Treatment with antiepileptic drugs is generally not indicated unless the seizures are particularly frequent and / or prolonged and this condition creates discomfort for the patient and family members; in $90 \%$ of cases there is a remission after a few years from the onset of the crises and especially from the age of 16. In some cases, the anomalies present in sleep are very represented so much that some authors speak of a spectrum that connects benign epilepsy with spikes (BECTS) to Landau-Kleffner syndrome.

e) Epilepsy with occipital paroxysms (CEOP form of 
Panayiotopoulos): Positive familiarity for epilepsy and intercritical EEG abnormalities in first degree relatives. Age of onset with a peak between 3 and 5 years. Crises typically occur at the beginning of sleep; critical episodes characterized by lateral deviation of the gaze and vomiting, often with an altered state of consciousness; crises can last for a long time. Many children no longer have epileptic seizures before they turn 10 while others can only have 1-2 episodes throughout their lives.

f) Landau-Kleffner syndrome (LKS): Also known as acquired epileptic aphasia, it is a rare, rapid-onset condition (in a previously normal child), characterized by symptoms that make the affected child appear "as if he were deaf"; a fluctuating and rapidly progressive course develops a disturbance in the understanding of language with the impossibility of decoding the meaning of some sounds (auditory agnosia, e.g. impossibility in understanding the meaning of a ringing telephone) and an expressive aphasia; other cognitive and behavioral problems may be present. The age of onset is between 3 and 8 years with a male / female ratio of 2:1. Generalized tonic-clonic crises, atypical absences, and focal motor crises can occur. The EEG shows frequent epileptic discharge especially during sleep and at the level of the temporal regions. It is an epileptic encephalopathy in which brain functioning is compromised by epileptic activity. The drugs most commonly used for treatment are corticosteroids and benzodiazepines; some children undergo epilepsy surgery.

g) Epilepsy with continuous wave-points in sleep or electric epileptic state in sleep (CSWS, ESES): The term electrical Epileptic State in Sleep (ESES) is synonymous with continuous wavespikes in sleep (CSWS); there is an overlap between epilepsy with continuous wave-spikes in sleep (CSWS) and Landau-Kleffner Syndrome (LKS) (the first is defined by EEG characteristics, while the second by clinical aspects); many children with LKS have a form of CSWS or a similar condition; LKS can be considered a type of CSWS with a temporal epileptogenic focus and consequent language regression. CSWS is characterized by the triad: continuous wave-spikes occupying more than $80 \%$ of slow-wave sleep, epileptic seizures, and cognitive-behavioral regression. The age of onset is typically between 4 and 6 years (range 1-11). Memory deficits, regression in cognitive functions, and hyperactivity may be present. Males are more affected than females. The first paroxysmal event is generally a generalized seizure in sleep (in some cases focal or focal crises with secondary generalization can occur which may have characteristics similar to the BECTS form). The evolution of this type of epilepsy is characterized by the possible appearance of other crises such as typical and atypical absences, myoclonic absences, clonic and atonic crises, generalized tonic-clonic crises. The treatment is based on the use of various antiepileptic drugs (especially benzodiazepines, valproic acid, ethosuximide or levetiracetam) and corticosteroids; carbamazepine can worsen critical symptoms; some children undergo epilepsy surgery.

h) Lennox-Gastaut syndrome (LGS): This condition defines a relatively rare and serious form of epilepsy characterized by the presence of tonic crises (always present element) or even atonic and atypical absence crises; in general, there is an identifiable symptomatic cause (in $30 \%$ of cases there is an "alleged symptomaticity"). The EEG is characterized by the presence of diffuse slow wave-points and rapid activity paroxysms. The prognosis for cognitive development, behavioral characteristics, and seizure control is negative.

i) Epilepsy with occipital paroxysms (CEOP form of Gastaut): Age of onset with a peak between 7 and 9 years. The seizures are characterized by brief visual symptoms without alteration of the state of consciousness and post-critical symptoms which include headache, nausea, and vomiting; some crises continue with versive type movements, sensory disturbances, automatisms, clones that are interesting to an emilate or widespread. This form of epilepsy compared to the Panayiotopoulos form has a slightly worse prognosis as regards the disappearance of the seizures. The EEG has characteristics similar to what is found in the form of Panayiotopoulos and presents as a characteristic element of epileptiform anomalies at the level of the occipital regions that are suppressed by opening the eyes ("fixation-off sensitivity") and activated by sleep.

\section{Adolescent onset epileptic syndromes}

a) Juvenile Absence Epilepsy (JAE): Age of onset with a peak at 12 years, typically near the pubertal period. Unlike the EWC there are few episodes of "absence" per day and the degree of impairment of the state of consciousness seems less even if the electrical anomalies tend to have a prolonged duration. About $80 \%$ of patients will have generalized tonic-clonic crises while $15 \%$ will also experience myoclonic crises (less intense than those that occur in juvenile myoclonic epilepsy). The EEG shows generalized anomalies consisting of $3 \mathrm{~Hz}$ wave-point complexes, often induced by hyperventilation; photosensitivity is unusual. Many patients respond to valproic acid treatment although the prognosis for the disappearance of long-term seizures is less good than for EWC.

b) Juvenile Myoclonic Epilepsy (JME): Debut between 12 and 18 years. The seizures are generalized and myoclonic tonic-clonic, and typically occur immediately after waking up; consciousness is preserved during myoclonic crises; generalized tonic-clonic crises are often preceded by a series of growing myoclonic crises; absence crises occur in about a third of cases. A story of objects falling from the hands while preparing breakfast is typical. Excessive tiredness, lack of sleep, and alcohol are potential trigger factors. The EEG typically shows discharges of poly points followed by irregular slow waves at a frequency between 1 and $3 \mathrm{~Hz}$; absence seizures have a correlated EEG of poly point-wave complexes at 4-6 $\mathrm{Hz}$ which slow down to $3 \mathrm{~Hz}$ (these epileptic discharges are much less regular than what is seen in the absence of epilepsy of the child or the epilepsy of absences of the youth). The antiepileptic drugs generally used are valproic acid, clonazepam, and levetiracetam; lamotrigine can cause an increase in myoclonic seizures but is effective in combination with valproic acid; carbamazepine treatment is contraindicated. The prognosis regarding seizure control is good but it is generally not recommended to stop anti-epileptic treatment due to the high risk of recurrence of crises in the absence of therapy.

Citation: Perrotta G (2020) Epilepsy: From pediatric to adulthood. Definition, classifications, neurobiological profiles and clinical treatments. J Neurol Neurol Sci Disord 6(1): 014-029. DOI: https://dx.doi.org/10.17352/jnnsd.000039 
c) Epilepsy with Generalized Tonic-Clonic Seizures (GTCS) upon awakening: Generally onset in the second decade of life. The diagnosis of this type of epilepsy is mainly clinical and should be suspected when generalized tonic-clonic seizures (GTCS) occur immediately after awakening, in the absence of frequent myoclonic hyperkinesias; seizures can be facilitated by reducing sleep hours; rather low seizure frequency. The prognosis is favorable both for the low frequency of the seizures and for the good response to the treatment (many patients have a reappearance of the seizures after the suspension of an effective antiepileptic treatment). Some patients who have presented other forms of epilepsy (e.g. CAE) in the past may have evolved in this condition. The EEG is typically characterized by the presence of irregular tip-wave or poly point-wave discharges with frequencies between 2 and $4 \mathrm{~Hz}$ (these alterations are not, however, found in all patients with routine EEG examinations; in this way case a sleeping EEG can provide additional diagnostic information); a fair percentage of patients have photosensitivity.

\section{Diagnostic profiles}

The diagnosis of epilepsy is typically formulated based on the description of the convulsive event and the circumstances in which it occurred, using an electroencephalogram and neuroimaging techniques for further study [38].

An Electroencephalogram (EEG) can help to show brain activity suggestive of an increased risk of experiencing convulsions (even during sleep), while resorting to biomedical imaging techniques, such as computed tomography and magnetic resonance imaging, it is recommended after a first non-febrile seizure to detect structural problems of the brain [39]. In particular [7]: in the Electroencephalogram (EEG) of an adult in normal conditions and a waking state, the alpha frequency represents the first and most common recordable signal. There is talk of an alpha wave, alpha rhythm, and alpha EEG:

1) Alpha rhythm: The number of waves in the alpha rhythm varies from 8 to 13 per second. In many individuals, the frequency is quite constant, and changes no more than 1-2 waves per second. In other cases, the alpha rhythm can vary from 8 to 14 waves without being considered an anomalous track. In normal individuals, an alpha rhythm is rarely observed which has more than 8 waves per second. The voltage of the seaweed wave, measured under normal conditions through the skull, records approximately 50-70 microvolts; occasionally it may be greater or lesser. During the recording made directly from the brain tissue, through the use of a thin metal electrode, the voltage of the maximum potential is very high, because the interferences and the dispersions of the skull and skin are eliminated. The alpha rhythm generally has a regular and continuous trend even if individual variations are possible. In some individuals the rhythm is very irregular, often disappears, or occurs only occasionally. This is not necessarily a symptom of serious brain disorders. The alpha rhythm can be recorded more precisely by electrodes placed in the posterior area of the skull where the brain region is located, which receives and processes signals from the retina. Considering the univocal relationship between the opening and closing of the eyes and the appearance of the alpha rhythm, it has been ascertained that the phenomena that concern the alpha are influenced by the stimuli that reach the brain passing through the retina and the optic nerve. When an individual psychically and physically in a state of rest opens his eyes, the voltage of the alpha rhythm is lowered and sometimes completely disappears. Normally, if the eyes remain open, it does not return regularly but appears occasionally for some time. In many individuals, the alpha rhythm can disappear if they undertake a mental activity such as that of making calculations. Although normally we speak of an alpha rhythm, it must be specified that a more careful observation of the traces can identify different brain areas from which it is possible to record as many alpha rhythms. The differences between these, however, are so small that they can safely speak of an alpha rhythm.

2) Beta rhythm: This variation in the brain's electrical potential is demonstrated by a large number of waves per second (frequency 14-40) but a very low voltage (about 2030 microvolts), about the already observed alpha rhythm. Beta rhythm occurs frequently, in small or large series of waves, and normally in sequences no longer than 1-2 seconds. It is normally recorded, as opposed to alpha rhythm, in the anterior region of the skull, i.e. at the front of the cerebral hemispheres. Beta rhythm is often observed in the body of some forms of brain activity for which intense concentration is required. If, for example, the patient is performing calculations, the replacement of the alpha rhythm with the beta rhythm frequently occurs.

3) Delta rhythm: The frequency of this electroencephalographic rhythm varies from 0 to 3.5 waves per second. In normal individuals, the frequency is never lower than two waves per second. The voltage is low and never exceeds 50 microvolts. This rhythm occurs irregularly, usually with isolated waves. The delta rhythm is usually recorded by electrodes located above the temporal lobes of the brain. The delta activity varies from individual to individual and often shows great differences even in the same individual if the electroencephalogram or EEG is recorded at different times.

4) Theta rhythm: This rhythm is characterized by waves having a frequency ranging from 4 to 7 per second, while for the voltage it generally does not exceed 50 microvolts. The theta rhythm presents itself with irregularities and is almost always recorded using electrodes placed above the temporal lobes of the brain.

A child's electroencephalogram differs in several features from that of an adult. From birth to around sixteen, the electroencephalogram changes evidently. During this period there are small and large variations. Immediately after birth, a limited activity of the EEG (electroencephalogram) can be observed, having a limited number of waves, from one to three per second, and a low voltage. In the period between 3 weeks and 3 months, the rhythm develops up to a frequency of 2-4 waves per second, and so also the deflection and the amplitude of the track. So the rhythm slowly reaches a frequency of 4-6 waves and later of 6-8 waves per second, measurable in most cases from the parietal bone and the occipital bone. 
The measurements are best obtained when the child keeps his eyes closed and the track is comparable to that of an adult alpha rhythm. During growth, the frequency increases until it reaches 8-10 waves per second, in children between 4 and 6 years of age, after which we can start talking about a normal and true alpha rhythm. The frequency continues to grow until it reaches the same measurable frequency in an adult at the age of ten. The whole of the EEG trace is not yet identical to that of an adult, since by recording with electrodes placed in the occipital area, it is possible to identify variations, the so-called alpha variants. These variants are waves in which the alpha rhythm contributes at the rate of one half, one-third of the overall frequency. During adolescent development, these alpha variants disappear and at 16 years the Electroencephalogram (EEG) is completely similar to that of an adult.

In epilepsy, the EEG records the spikes, poly points and wave-point complexes in an anomalous way; in particular, it records the so-called peak wave systems or complexes, with a frequency of 3 waves per second and a high voltage of 250-400 microvolts, with peaks of frequencies between 10-20 waves, always at high voltage.

In adults, again, from a diagnostic point of view, the blood values of electrolytes, blood sugar, and calcium levels are important to exclude some pathologies as a possible cause of the epileptic episode, while an electrocardiogram can exclude problems of the rhythm of the heart. Finally, a lumbar puncture can help diagnose an infection of the central nervous system, but it is not usually not done routinely. In children, further tests may be required such as urine and blood tests for possible metabolic disorders. An elevated blood level of prolactin, for example, within the first twenty minutes after an attack, can be useful to confirm an epileptic situation as opposed to the nonepileptic psychogenic seizure. However, the serum prolactin level is less useful for identifying partial seizures (Figures 1.11.4) $[40-46]$.

\section{Clinical treatments}

Epilepsy is usually treated through the daily intake of drugs, prescribed after the occurrence of a second seizure treated mainly in the hospital setting. During first aid, it is essential to put people with an active tonic-clonic seizure in the lateral safety position to help prevent inhalation of fluids in the lungs. Putting your fingers in your mouth or inserting a tongue depressor is not recommended as it may cause vomiting or cause the rescuer to be bitten. The interventions should aim to avoid trauma, however, precautions for the spine are generally not necessary. If an attack lasts longer than 5 minutes or there are two attacks within an hour without a return to a normal level of consciousness in between, there is a belief that there is a medical emergency known as a "state of illness epileptic". This situation may need medical attention to keep the airway patency protected. The most commonly used drug for a longterm attack is midazolam per os, while diazepam can also be administered rectally. Intravenous lorazepam is preferred in the hospital. If two doses of benzodiazepines are not effective, other drugs such as phenytoin can be used. Seizures that do not respond to initial treatment typically require hospitalization in an intensive care unit and treatment with major medications such as thiopental sodium or propofol. Anticonvulsant drugs are the main treatment for epilepsy and often have to be taken for life. The choice of the active ingredient is based on the type of seizure, on the presence of epilepsy syndrome, on the other drugs prescribed, on the other health problems, and the age and lifestyle of the person. Initially, a single drug is recommended and if this is not effective, or involves serious side effects, you try to change. Taking two drugs at the same time is only recommended if the single does not provide results. In about

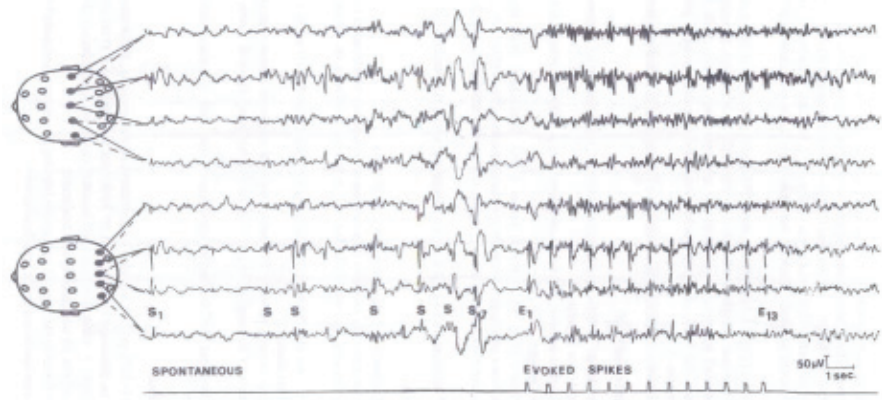

Figure 1.1: Epilepsy with spontaneous parasagittal parietal tips (S1-S7) and evoked by sensory stimuli (E1-E13) [45].

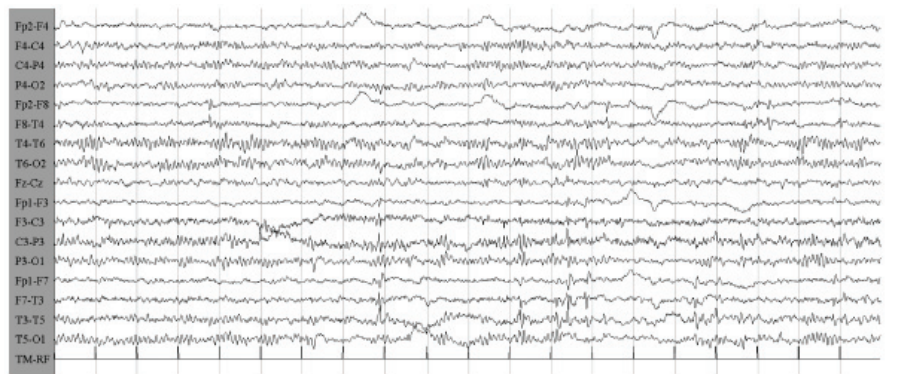

Figura 10

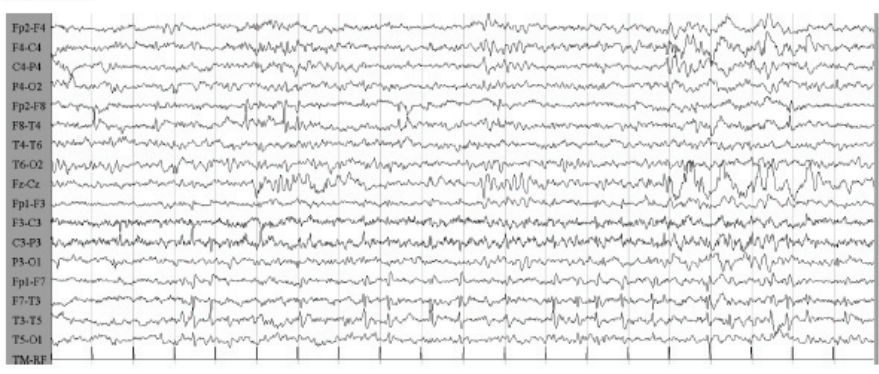

Figure 1.2: Benign childhood epilepsy with centrotemporal spikes (BECTS) or rolandic epilepsy [46].

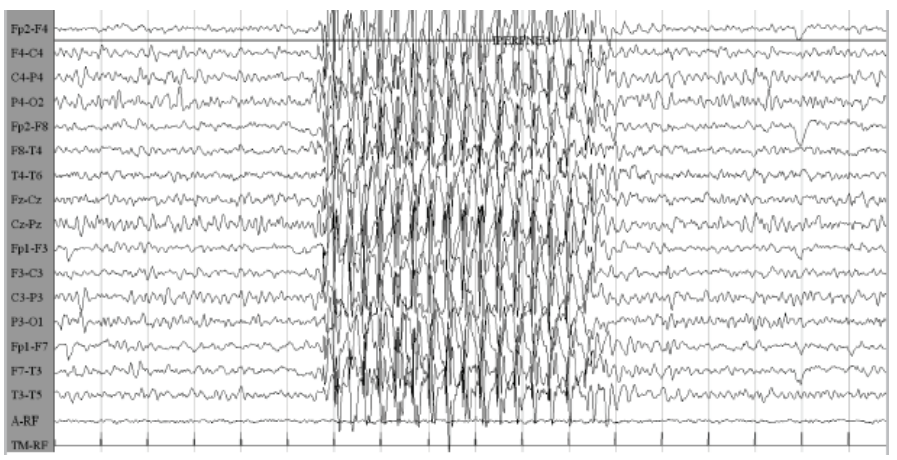

Figure 1.3: Absence type epilepsy in children [46]. 


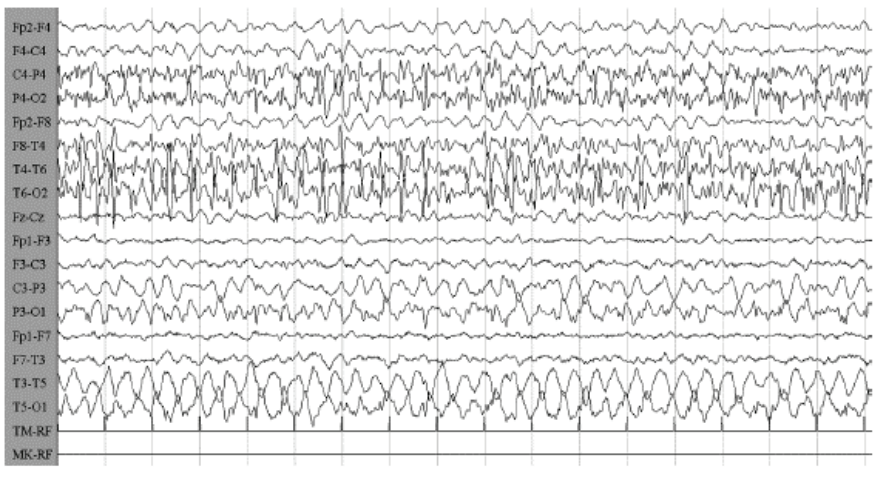

Figure 1.4: Symptomatic focal epilepsy of Sturge-Weber syndrome [46]

half, the first prescription is already effective. There are also several drugs available that seem to be equally effective, both for partial and generalized seizures: phenytoin, carbamazepine, and valproate. Controlled-release carbamazepine appears to work like immediate-release carbamazepine and may have fewer side effects. For generalized seizures, valproate is the recommended drug and lamotrigine can be considered the second choice. In those who experience a seizure of absence, ethosuximide or valproate are recommended; the latter is particularly effective in myoclonic and tonic seizures or unstressed convulsions. If the attacks appear well controlled following a particular treatment, it is generally not necessary to regularly check the drug levels in the blood. In the case of drug-resistant epilepsy, resorting to surgery may be an option for individuals with partial epileptic seizures that do not cease to manifest despite the adoption of other treatments. Before evaluating surgical treatment, however, it is necessary to try at least two or three different drugs. The purpose of surgery is the total control of epileptic seizures. Common procedures include cutting the hippocampus through an anterior temporal lobe resection, removing tumor masses, and removing portions of the neocortex. Some procedures such as callosotomy may be attempted to try to reduce the number of seizures, rather than to cure the condition itself. Following surgery, in many cases, drug therapy can be slowly stopped. Neurostimulation may also be a viable option in those who are not candidates for surgery. Three methods have proven to be effective in those who do not respond to drugs: vagus nerve stimulation, anterior thalamic stimulation, and closed-loop response stimulation. Finally, a ketogenic diet (high-fat content, low carbohydrate content, adequate proteins) seems to decrease the number of attacks by half in about $30-40 \%$ of children [47-57].

\section{Conclusions}

Epilepsy often has negative effects on social and psychological well-being. These effects may include social isolation, stigmatization or a real state of disability, poor academic performance, and on average lower occupations, but also learning difficulties, attention disturbances, and socialization problems. All aspects that deserve to be adequately addressed with the help of a psychotherapist, supported by the clinical staff. Progress in research is giving encouraging signals and new, less and less invasive, targeted therapies are passing the test of scientific reliability. It is assumed, in the recent future, that attention will be increasingly focused on the well-being of patients and their definitive recovery.

\section{References}

1. Magiorkinis E, Kalliopi S, Diamantis A (2010) Hallmarks in the history of epilepsy: epilepsy in antiquity. Epilepsy Behav 17: 103-108. Link: https://bit.ly/2CPDeYO

2. Fisher RS, Acevedo C, Arzimanoglou A, Bogacz A, Cross JH, et al. (2014) Una definizione clinico-pratica di epilessia (adattamento in italiano). Epilepsia 55: 475-482. Link: https://bit.ly/3jINsRD

3. Chang BS, Lowenstein DH (2003) Epilepsy. N Engl J Med 349: 1257-1266. Link: https://bit.ly/3fJ3hzo

4. Fisher R, Acevedo C, Arzimanoglou A, Bogacz A, Cross JH, et al. (2014) ILAE Official Report: A practical clinical definition of epilepsy. Epilepsia 55: 475-482. Link: https://bit.ly/20IAwa7

5. Fisher R, Emde Boas W, Blume W, Elger C, Genton P, et al. (2005) Epileptic seizures and epilepsy: definitions proposed by the International League Against Epilepsy (ILAE) and the International Bureau for Epilepsy (IBE). Epilepsia 46: 470-472. Link: https://bit.ly/2E57Q9m

6. Perrotta G (2019) Psicologia clinica. Luxco ed., I ed.

7. Perrotta G (2019) Psicologia generale. Luxco ed., I ed.

8. Scheffer IE, Berkovic S, Capovilla G, Connolly MB, French J, et al. (2017) Classificazione delle epilessie della International League Against Epilepsy: position paper della Commissione ILAE per la Classificazione e la Terminologia. Epilepsia 58: 512-521. Link: https://bit.ly/3fOvqVS

9. Pandolfo M (2011) Genetics of epilepsy. Semin Neurol 31: 506-518. Link: https://bit.ly/3eUmaho

10. Kumar D (2008) Genomics and clinical medicine. Oxford, Oxford University Press, 279. Link: https://bit.ly/2E5OINV

11. Berg AT, Berkovic SF, Brodie MJ, Buchhalter J, Cross JH, et al. (2010) Revised terminology and concepts for organization of seizures and epilepsies: report of the ILAE Commission on Classification and Terminology, 2005- 2009. Epilepsia 51: 676-685. Link: https://bit.ly/2OP5ZY9

12. Longo DL (2012) Seizures and Epilepsy. In Harrison's principles of interna medicine. $18^{\text {th }}$, McGraw-Hill 3258.

13. Berkovic SF, et al. (2006) Human epilepsies: interaction of genetic and acquired factors. Trends Neurosci 29: 391-397. Link: https://bit.ly/2OLgSu5

14. Neligan A, Hauser WA, Sander JW (2012) The epidemiology of the epilepsies. Handbook of clinical neurology 107: 113-133. Link: https://bit.ly/2CpRrvT

15. Eadie MJ (2012) Shortcomings in the current treatment of epilepsy. Expert Rev Neurother 12: 1419-1427. Link: https://bit.ly/3jrvFsc

16. Hauser WA, Kurland LT (1967) The epidemiology of epilepsy in Rochester Minnesota, 1935 through 1967. Epilepsia 16: 1-66. Link: https://bit.ly/39hKOCP

17. Sander JW (2003) The epidemiology of epilepsy revisited. Curr Opin Neurol 16 165-170. Link: https://bit.ly/20Fdilo

18. Thurman DJ, Beghi E, Begley CE, Berg AT, Buchhalter JR, et al. (2011) Standards for epidemiologic studies and surveillance of epilepsy. Epilepsia 52: 2-26. Link: https://bit.ly/3eP6XhE

19. Collaborators GBD 2013 Mortality and Causes of Death (2015) Global, regional, and national age-sex specific all-cause and cause-specific mortality for 240 causes of death, 1990-2013: a systematic analysis for the Global Burden of Disease Study 2013. Lancet 385: 117-1171. Link: https://bit.ly/3eK3W2p 
20. Blumenfeld H (2005) Cellular and Network Mechanisms of Spike-Wave Seizures. Epilepsia 46: 21-33. Link: https://bit.ly/20GVK8f

21. Quyen MV, Navarro V, Martinerie J, Baulac J, Varela FJ (2003) Toward a Neurodynamical Understanding of Ictogenesis. Epilepsia 44: 30-43. Link: https://bit.ly/2Biwy57

22. da Silva FL, Blanes W, Kalitzin SN, Parra J, Suffczynski P, et al. (2003) Epilepsies as Dynamical Diseases of Brain Systems: Basic Models of the Transition Between Normal and Epileptic Activity. Epilepsia 44: 72-83. Link: https://bit.ly/3fP9ABo

23. Goldberg EM, Coulter DA (2013) Mechanisms of epileptogenesis: a convergence on neural circuit dysfunction. Nat Rev Neurosci 14: 337 349. Link: https://bit.ly/2ZNx8B7

24. Oby E, Janigro D (2006) The blood-brain barrier and epilepsy. Epilepsia 47: 1761-1774. Link: https://bit.ly/2ZLsy6n

25. Somjen GG (2004) Ions in the Brain Normal Function, Seizures, and Stroke., New York, Oxford University Press 167. Link: https://bit.ly/2OIMH6T

26. Engel J, Pedley TA (2008) Epilepsy : a comprehensive textbook. $2^{\text {nd }}$ ed. Philadelphia, Wolters Kluwer Health/Lippincott Williams \& Wilkins, 483. Link: https://bit.ly/3fODy80

27. Angelini C, Battistin L (2014) Neurologia clinica. Società editrice Esculapio.

28. Perrotta G (2019) La possessione demoniaca. Riedizione, Luxco ed.

29. Perrotta G (2019) Psicologia generale. Luxco ed., I ed

30. Bradley WG (2012) Bradley's neurology in clinical practice. $6^{\text {th }}$ ed., Philadelphia, PA, Elsevier/Saunders

31. Panayiotopoulos CP (2010) A clinical guide to epileptic syndromes and their treatment based on the ILAE classifications and practice paramete guidelines. Rev $2^{\text {nd }}$ ed., London, Springer 445

32. Wheless JW (2009) Advanced therapy in epilepsy, Shelton, Conn., People's Medical Pub. House 443

33. Larner AJ (2010) A dictionary of neurological signs. $3^{\text {rd }}$ ed., New York Springer 348.

34. Schachter SC (2008) Behavioral aspects of Epilepsy: principles and practice New York, Demos 125. Link: https://bit.ly/2DWKYZs

35. Xue LY, Ritaccio AL (2006) Reflex seizures and reflex epilepsy. Am J Electroneurodiagnostic Technol 46: 39-48. Link: https://bit.ly/2ZKuJr4

36. Malow BA (2005) Sleep and epilepsy. Neurol Clin 23: 1127-1147. Link: https://bit.ly/2COklp7

37. Tricomi G (2014) Le epilessie in età pediatrica: inquadramento diagnostico. Quaderni ACP 21: 100-109.

38. National Institute for Health and Clinical Excellence, Chapter 1: Introduction (PDF) (2012) The Epilepsies: The diagnosis and management of the epilepsies in adults and children in primary and secondary care. National Clinical Guideline Centre, gennaio 21-28. Link: https://bit.ly/2BgY8j1

39. National Institute for Health and Clinical Excellence, Chapter 4: Guidance (PDF), In The Epilepsies: The diagnosis and management of the epilepsies in adults and children in primary and secondary care. National Clinical Guideline Centre, gennaio 57-83. Link: https://bit.ly/2BgY8j1

40. Wilden JA, Cohen-Gadol AA (2012) Evaluation of first nonfebrile seizures American family physician. 86: 334-340. Link: https://bit.ly/3g0PQv3

41. Wallace SJ, Farrell K (2004) Epilepsy in children. $2^{\text {nd }}$ ed, London, Arnold 354.

42. Luef G (2010) Hormonal alterations following seizures. Epilepsy \& Behavio E\&B 19: 131-133. Link: https://bit.ly/2WEfL3G

43. Ahmad S, Beckett MW (2004) Value of serum prolactin in the management of syncope. Emerg Med J 21: e3. Link: https://bit.ly/3fMl4FS

44. Shukla G, Bhatia M, Vivekanandhan S, Gupta N, Tripathi M, et al. (2004) Serum prolactin levels for differentiation of nonepileptic versus true seizures: limited utility. Epilepsy Behav E\&B 5: 517-521. Link: https://bit.ly/2ZOaOYO

45. Marco P, Tassinari CA (1981) Extreme Somatosensory Evoked Potentia (ESEP): An EEG Sign Forecasting the Possible Occurrence of Seizures in Children. Epilepsia 22: 569-575. Link: https://bit.ly/39h820g

46. Giovanni T (2014) Le epilessie in età pediatrica: inquadramento diagnostico Quaderni ACP 21: 100-109. Link: https://bit.ly/2ZQrSNf

47. Michael GE, O'Connor RE (2011) The diagnosis and management of seizures and status epilepticus in the prehospital setting. Emerg Med Clin North Am 29 29-39. Link: https://bit.ly/2CTXubT

48. Wyllie E (2012) Wyllie's Treatment of Epilepsy: Principles and Practice Lippincott Williams \& Wilkins, 187

49. Nolan SJ, Marson AG, Weston J, Smith CT (2013) Phenytoin versus valproate monotherapy for partial onset seizures and generalised onset tonic-clonic seizures. Cochrane Database Syst Rev 8: CD001769. Link: https://bit.ly/2WFXfYY

50. Smith CT, Marson AG, Clough HE, Williamson PR (2002) Carbamazepine versus phenytoin monotherapy for epilepsy. Cochrane Database Syst Rev CD001911. Link: https://bit.ly/39gEYq5

51. Powell G, Saunders M, Marson AG (2010) Immediate-release versus controlledrelease carbamazepine in the treatment of epilepsy. Cochrane Database Syst Rev 12: CD007124. Link: https://bit.ly/39dxLqX

52. Ilangaratne NB, Mannakkara NN, Bell GS, Sander JW (2012) Phenobarbital: missing in action. In Bulletin of the World Health Organization 90: 871-871. Link: https://bit.ly/2Bh08I3

53. Perucca P, Gilliam FG (2012) Adverse effects of antiepileptic drugs. Lancet Neurol 11: 792-802. Link: https://bit.ly/39g5G1Z

54. Kamyar M, Varner M (2013) Epilepsy in pregnancy. Clin Obstet Gynecol 56: 330-341. Link: https://bit.ly/2ZOclb6

55. Bergey GK (2013) Neurostimulation in the treatment of epilepsy. In Experimental neurology 244: 87-95.

56. Levy RG, Cooper PN, Giri P (2012) Ketogenic diet and other dietary treatments for epilepsy. In The Cochrane database of systematic reviews 3 : CD001903. Link: https://bit.ly/39mU6ST

57. Noebels JL, Avoli M (2012) Jasper's Basic Mechanisms of the Epilepsies, Oxford University Press 29: 466. Link: https://bit.ly/39e1YGa

Copyright: (C) 2020 Perrotta G. This is an open-access article distributed under the terms of the Creative Commons Attribution License, which permits unrestricted use distribution, and reproduction in any medium, provided the original author and source are credited.

Citation: Perrotta G (2020) Epilepsy: From pediatric to adulthood. Definition, classifications, neurobiological profiles and clinical treatments. J Neurol Neurol Sci Disord 6(1): 014-029. DOI: https://dx.doi.org/10.17352/jnnsd.000039 